\title{
CDK5RAP3 Deficiency Restrains Liver Regeneration after Partial Hepatectomy Triggering Endoplasmic Reticulum Stress
}

\author{
Shuchun Yang, ${ }^{* \dagger}$ Rui Yang, ${ }^{{ }^{\dagger}}$ Huanmin Wang, ${ }^{* \dagger}$ Yue Huang, ${ }^{* \dagger}$ and Yuyan Jia ${ }^{* \dagger}$
}

From the State Key Laboratory of Medical Molecular Biology* and the Department of Medical Genetics, ${ }^{\dagger}$ Institute of Basic Medical Sciences, Chinese Academy of Medical Sciences and Peking Union Medical College, Beijing, China

\author{
Accepted for publication \\ August 24, 2020. \\ Address correspondence to \\ Yuyan Jia, Ph.D., or Yue \\ Huang, Ph.D., Department of \\ Medical Genetics, Institute of \\ Basic Medical Sciences, Chi- \\ nese Academy of Medical Sci- \\ ences \& Peking Union Medical \\ College, Dong Dan San Tiao 5, \\ Beijing 100005. E-mail: \\ jiayuyan@ibms.pumc.edu.cn or \\ huangyue@pumc.edu.cn.
}

\begin{abstract}
CDK5 regulatory subunit-associated protein 3 (CDK5RAP3) plays a crucial role in mammalian liver development and hepatic function by controlling hepatocyte proliferation and differentiation, glucose and lipid metabolism, UFMylation, and endoplasmic reticulum homeostasis. However, the role of CDK5RAP3 in liver regeneration remains unknown. A liver-specific C $d k 5$ rap 3 knockout (CKO) mouse model was used to study the function of CDK5RAP3 during liver regeneration induced by standard two-thirds partial hepatectomy (PHx). Twenty-four hours after $\mathrm{PHx}$, the liver-to-body weight ratio was markedly higher in CKO mice than in wild-type mice. However, this ratio did not increase significantly and gradually over time after PHx in CKO mice. Hepatocyte proliferation was significantly delayed in CKO mice compared with wild-type mice. Meanwhile, CDK5RAP3 deficiency increased lipid accumulation, impaired glycogen synthesis, and lowered blood glucose levels after PHx. Critically, the absence of CDK5RAP3 seemed to promote an inflammatory response and induce apoptosis at a late stage of liver regeneration. In addition, CDK5RAP3 deficiency disrupted UFMylation homeostasis and aggravated endoplasmic reticulum stress in hepatocytes after PHx. Taken together, these data suggest that CDK5RAP3 enhances liver regeneration, at least partially via controlling cell cycle and glucose and lipid metabolism. (Am J Pathol 2020, 190: 2403-2416; https://doi.org/10.1016/j.ajpath.2020.08.011)
\end{abstract}

The liver has a unique capacity to regenerate after various types of injuries. ${ }^{1,2}$ However, patients with acute liver failure or who undergo extensive liver resection frequently experience failed regeneration. ${ }^{3,4}$ Thus, it is critical to better elucidate the mechanisms underlying liver homeostasis and regeneration, and to identify new therapeutic targets that can regulate liver regeneration. This tightly modulated process involves priming of the hepatocytes, cell cycle entry, proliferation, and termination., Two-thirds partial hepatectomy (PHx) in rodents is a common model to investigate liver regeneration. ${ }^{7}$ After $\mathrm{PHx}$, the residual hepatocytes replicate through both hypertrophic and hyperplastic mechanisms. ${ }^{8,9}$ Liver regeneration relies primarily on hepatocyte proliferation. ${ }^{10}$ Upon $\mathrm{PHx}$, quiescent hepatocytes quickly reenter the cell cycle, ${ }^{11}$ with cell proliferation peaking at day 2 or $3 .^{12-14}$ High amounts of lipids and glycogen accumulate in hepatocytes immediately after two-thirds $\mathrm{PHx} .{ }^{8}$ Transient liver steatosis on day 2 or 3 after $\mathrm{PHx}$ influences liver regeneration. ${ }^{15,16}$ Lipid overload in mice with simple hepatic steatosis can delay DNA replication in hepatocytes during liver regeneration. ${ }^{17}$

CDK5RAP3 (or LZAP, C53) was first identified as a binding protein for cyclin-dependent kinase 5 activator. ${ }^{18,19}$ CDK5RAP3 is highly conserved in mammals and is expressed in multiple organs, especially in liver. ${ }^{18-20}$ CDK5RAP3 interacts with several proteins, including P35, Rel A, Chk1, TIP-1, and CBP, to regulate cell cycle, cell survival, cell adherence/invasion, tumorigenesis, and metastasis. $^{21-25}$ In addition, CDK5RAP3 participates in maintenance of protein homeostasis by regulating endoplasmic

Supported by Chinese Academy of Medical Sciences Innovation Fund for Medical Sciences grant 2017-I2M-1-008 (Y.J.), National Key Research and Development Program of China grant 2016YFA0100103 (Y.H.), and National Natural Science Foundation of China grant 31501176 (Y.J.).

Disclosures: None declared. 
reticulum (ER) homeostasis and UFMylation, the latest ubiquitylation-like modification identified. ${ }^{20,26,27}$

Ubiquitin-fold modifier 1 (UFM1) is the most recently identified ubiquitin-like protein. ${ }^{28}$ The UFM1 conjugation system comprises UFM1, UBA5 (E1 activating enzyme), UFC1 (E2 conjugating enzyme), UFL1 (E3 ligase), and UFSP1 and UFSP2 (UFM1-specific proteases). ${ }^{29-31}$ CDK5RAP3 has been identified as a substrate for $\mathrm{UFM}^{32}$ and can interact with UFC1 and UFL1. ${ }^{27,33}$ A growing body of evidence implicates the UFMylation system in many biological processes, including hematopoiesis, brain and liver development, ER stress, and fatty acid metabolism. ${ }^{20,34}$ However, the function of the UFMylation system in liver regeneration remains largely unknown and warrants further investigation.

Here, the function of CDK5RAP3 in liver regeneration was explored in the most commonly used two-thirds PHx mice model. ${ }^{35}$ We found that CDK5RAP3 deficiency does not completely abolish but dramatically impairs liver regeneration, as indicated by delays in hepatocyte proliferation, lipid accumulation, and impaired glycogen synthesis in remnant livers. In regenerating livers, CDK5RAP3 deficiency perturbs UFMylation homeostasis and aggravates ER stress. Taken together, these data indicate that CDK5RAP3 plays a significant role in liver regeneration by regulating hepatocyte proliferation and glucolipid metabolism.

\section{Materials and Methods}

Mice

Alb-Cre induced liver-specific Cdk5rap3 knock-out (CKO) mice were generated, as previously described. ${ }^{20}$ The mice were kept with an alternating 12-hour light/ dark cycle and provided with standard chow and water ad libitum. The Institutional Animal Care and Use Committee at Peking Union Medical College \& Chinese Academy of Medical Sciences approved all mice procedures. All mice were fed in specific pathogen-free facilities.

\section{PHx Model}

The two-thirds PHx was performed on 2- to 3-month-old C57BL6/J mice as previously reported. ${ }^{36,37}$ Bromodeoxyuridine (BrdU) $(50 \mathrm{mg} / \mathrm{kg}$ body weight) was injected intraperitoneally into mice 2 hours before euthanasia at the indicated time points.

\section{Immunohistochemistry and Western Blot}

Paraffin-embedded liver tissues were cut into sections for hematoxylin and eosin (H\&E) and immunohistochemistry staining. Optimal cutting temperature-embedded blocks were cut into sections for Oil Red $\mathrm{O}$ staining. Whole liver protein extracts were obtained by homogenizing liver samples in a radioimmunoprecipitation assay buffer (Beyotime, Beijing, China) and protease/phosphatase inhibitors. H\&E staining, immunohistochemistry staining, periodic acid-Schiff staining, Oil Red O staining, and Western blot were performed as previously reported. ${ }^{20}$

\section{Antibodies}

Primary antibodies were used against $\beta$-catenin (Santa Cruz Biotechnology, Dallas, TX), matrix metallopeptidase 2 ( Santa Cruz Biotechnology), P21 (Abcam, Cambridge, UK), $\beta$-actin (Santa Cruz Biotechnology), BrdU (Abcam), CDK5RAP3 (Abcam, Cambridge, UK), Chop (Abcam), glyceraldehyde-3-phosphate dehydrogenase (Santa Cruz Biotechnology), proliferation cell nuclear antigen (PCNA) (Santa Cruz Biotechnology), pHH3 (Abcam), p-PERK (Cell Signaling Technology, Boston, MA), activating transcription factor 6 (Cell Signaling Technology), XBP1 (Abcam), IRE1 $\alpha$ (Santa Cruz Biotechnology), activating transcription factor 4 (Abcam), p-eIF2 $\alpha$ (Cell Signaling Technology), CyclinB1 (Santa Cruz Biotechnology), CyclinD1 (Abcam), Bip (Cell Signaling Technology), Ufm1 (Abcam), and active caspase-3 (R\&D system, Minneapolis, MN). All horseradish peroxidase-linked secondary antibodies were purchased from Cell Signaling Technology.

\section{RNA Extraction and Real-Time Quantitative PCR}

Total RNA was extracted from liver tissues in various time points after PHx using Trizol reagent (Thermo Fisher Scientific, Waltham, MA). Reverse transcription and realtime quantitative PCR were performed as previously described. $^{20} \beta$-actin expression was used to normalize gene expression. The sequences of primers are given in Table 1.

\section{Serum Biochemical Analysis}

After at least 30 minutes of coagulation at room temperature and centrifugation, serum was separated and stored at $-80^{\circ} \mathrm{C}$. Hitachi 7100 Automatic Analyzer (Hitachi, Tokyo, Japan) was used to determine serum levels of cholesterol, triglycerides, total bilirubin, alanine aminotransferase, and aspartate aminotransferase. The OneTouch Ultra System Kit (BD Biosciences, San Jose, CA) was used to measure the serum level of glucose. 
Table 1 Primer Sequences of Real-Time Quantitative PCR Used in This Study

\begin{tabular}{ll}
\hline Name & Sequence \\
\hline Cd36-F & $5^{\prime}$-ATGGGCTGTGATCGGAACTG-3' \\
Cd36-R & $5^{\prime}$-TTTGCCACGTCATCTGGGTTT-3' \\
Cpt1 $\alpha-\mathrm{F}$ & $5^{\prime}$-TGGCATCATCACTGGTGTGTT-3' \\
Cpt1 $\alpha-\mathrm{R}$ & $5^{\prime}$-GTCTAGGGTCCGATTGATCTTTG-3' \\
Hadha-F & $5^{\prime}$-TGCATTTGCCGCAGCTTTAC-3' \\
Hadha-R & $5^{\prime}$-GTTGGCCCAGATTTCGTTCA-3' \\
Hadhb-F & $5^{\prime}$-TCGGGTTTGTTGCATCGGA-3' \\
Hadhb-R & $5^{\prime}$-GGCCAGAAGCTATCAGACCAA-3' \\
Scd1-F & $5^{\prime}$-TTCTTGCGATACACTCTGGTGC-3' \\
Scd1-R & $5^{\prime}$-CGGGATTGAATGTTCTTGTCGT-3' \\
Acaca-F & $5^{\prime}$-CTCCCGATTCATAATTGGGTCTG-3' \\
Acaca-R & $5^{\prime}$-TCGACCTTGTTTTACTAGGTGC-3' \\
Fasn-F & $5^{\prime}$-GGAGGTGGTGATAGCCGGTAT-3' \\
Fasn-R & $5^{\prime}$-TGGGTAATCCATAGAGCCCAG-3' \\
Cdk5rap3-F & $5^{\prime}$-CAACACTGCCATCCAGGACAT-3' \\
Cdk5rap3-R & $5^{\prime}$-GCCTCGGTGCCTTTAAGAAG-3' \\
$\beta$-actin-F & $5^{\prime}$-ACCAACTGGGACGACATGGAGAAG-3' \\
$\beta$-actin-R & $5^{\prime}$-TACGACCAGAGGCATACAGGGACA-3' \\
\hline
\end{tabular}

$F$, forward; $R$, reverse.

\section{Statistical Analysis}

Results are presented as means \pm SD. Data were assessed by using a $t$-test. Differences were considered statistically significant at $P<0.05$. Prism 7.0 (GraphPad Software, La Jolla, CA) was used to perform statistical analyses.

\section{Results}

\section{Cdk5rap3 Deletion Causes Aberrant Liver Regeneration after $\mathrm{PHx}$}

CDK5RAP3 reportedly regulates cell proliferation and differentiation $^{23-25}$ and plays a crucial role in liver development. ${ }^{20}$ However, the function of CDK5RAP3 in liver regeneration remains unknown. To investigate the kinetics of CDK5RAP3 expression during liver regeneration, wild-type (WT) mice underwent two-thirds PHx or sham surgery, and were euthanized at different time points after surgery. CDK5RAP3 expression level did not vary significantly during this process (Figure 1A). Cyclin D1 expression was dramatically increased at 24, 36, and 48 hours after PHx (Figure 1A). Meanwhile, the liver-tobody weight $(\mathrm{LW} / \mathrm{BW})$ ratio increased significantly from 24 hours after PHx onward (Supplemental Figure S1A). The number of PCNA-positive hepatocytes also dramatically increased at 24, 36, and 48 hours after $\mathrm{PHx}$ (Supplemental Figure S1B).

To further investigate the role of CDK5RAP3 in liver regeneration, CKO mice were studied. In addition, the Cre-negative littermates were used as WT controls. CKO mice displayed no obvious defects and survived well into adulthood, possibly because of incomplete deletion of Cdk5rap 3 in hepatocytes at early developmental stages. ${ }^{20}$ CKO mice had an LW/BW ratio comparable to that of WT mice, although there was high efficiency of Cdk5rap3 deletion in adult mice (Figure 1, B-D, and Supplemental Figure S1C). H\&E staining showed that the hepatocytes in CKO mice were slightly larger than in WT mice (Figure 1B). Meanwhile, lipid accumulation measured by using Oil Red $\mathrm{O}$ staining was slightly increased in CKO mice (Figure 1B). There were still a few CDK5RAP3-positive hepatocytes in CKO due to leaky Cre (Figure 1B).

Next, CKO and WT adult mice underwent two-thirds PHx. The LW/BW ratio was markedly increased at 24 hours after PHx in both WT and CKO mice (Figure 1D). Interestingly, the LW/BW ratio at 24 hours after PHx was significantly higher in CKO mice than in WT mice (Figure 1D). After PHx, the LW/BW ratio of WT mice increased gradually and significantly over time, contrary to the LW/BW ratio in CKO mice (Figure 1D). Sham surgery did not affect survival of CKO mice, and the LW/BW ratio did not statistically differ between WT and KO mice subjected to sham operation at the time points studied (Supplemental Figure S1D). In addition, the level of serum alanine aminotransferase, indicative of the extent of liver damage, was elevated more in CKO mice than in WT mice (Figure 1E).

To further assess the consequence of liver CDK5RAP3 deficiency on proliferation, cell cycle, and inflammation, the expression of the proteins $\beta$-catenin, cyclin D1, matrix metallopeptidase 2, and P21 was measured after PHx. The expression of $\beta$-catenin and cyclin D1 was significantly decreased in CKO mice compared with WT mice, whereas matrix metallopeptidase 2 and P21 levels were up-regulated in CKO mice (Figure 1, F and G). Collectively, these results suggest that Cdk5rap3 deletion leads to aberrant liver regeneration after PHx.

\section{CDK5RAP3 Deficiency Impairs Hepatocyte Proliferation after $\mathrm{PHx}$}

To assess whether liver-specific Cdk5rap3 knockout suppressed hepatocyte proliferation after PHx, PCNA expression and BrdU incorporation were measured. Unlike in WT mice, PCNA protein levels did not increase markedly at 24 hours after PHx in CKO livers (Figure 2A). In CKO mice, expression of PCNA was not up-regulated until 48 hours after PHx (Figure 2A). The number of PCNA- and BrdU-positive hepatocytes was also decreased in CKO mice compared with control mice (Figure 2, B and $\mathrm{C}$ ). Considering previous reports showing that Cdk5rap3 deficiency led to $\mathrm{G} 2 / \mathrm{M}$ arrest, ${ }^{20}$ cell cycle progression was evaluated by measuring the level of pHH3. At 36 and 48 hours after PHx, the 
A

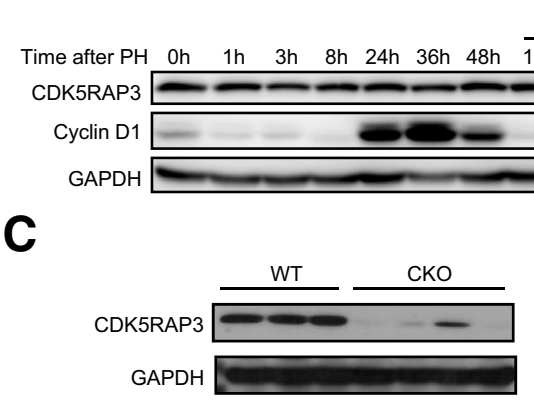

B

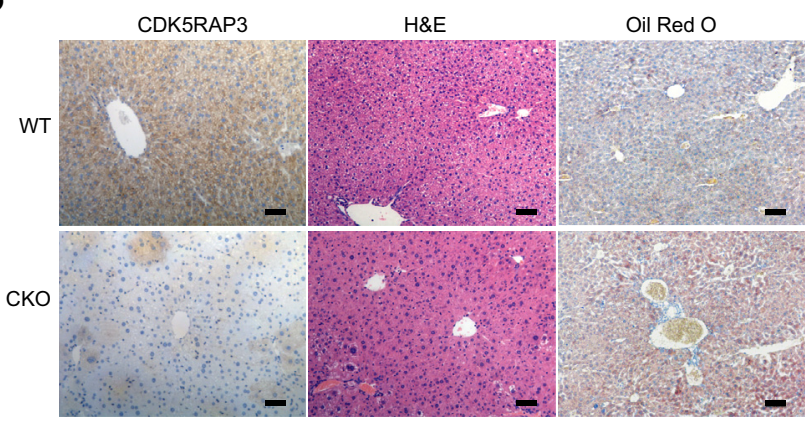

$\mathbf{E}$

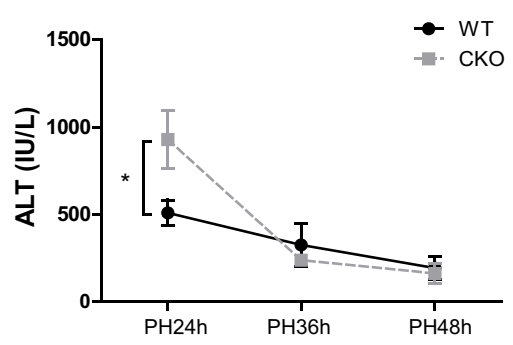

$\mathbf{F}$
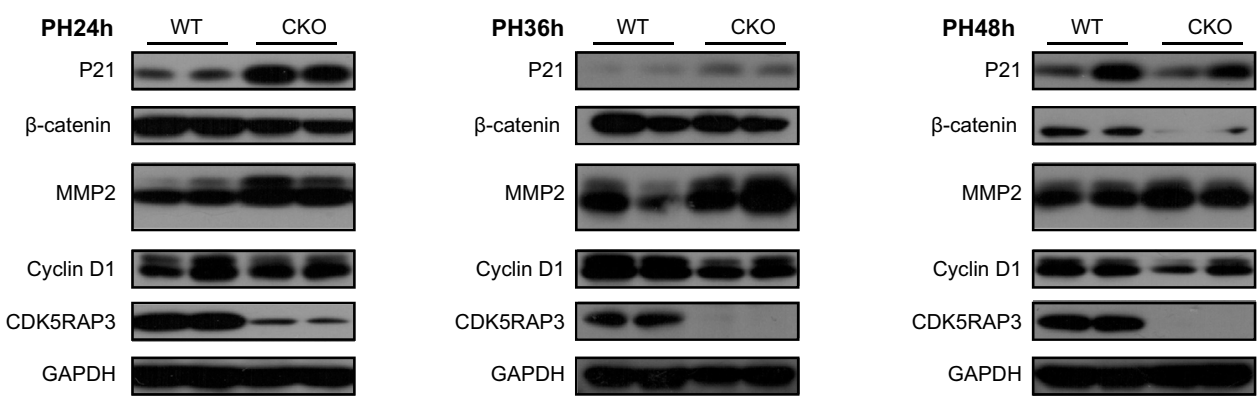

G
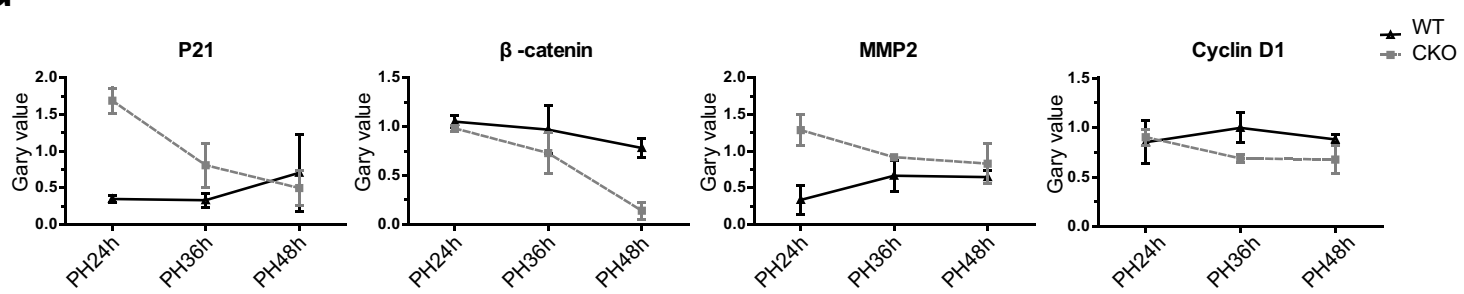

Figure 1 CDK5 regulatory subunit-associated protein 3 (CDK5RAP3) deletion impairs liver regeneration after partial hepatectomy (PHx). A: Expression of CDK5RAP3 and cyclin D1 in wild-type (WT) livers was determined by using Western blot analysis, 0 to 48 hours after PHx and 1 to 3 hours after sham surgery. B: Representative images of immunohistochemistry staining for CDK5RAP3, and hematoxylin and eosin (H\&E) staining and 0il Red 0 staining of WT and liver-specific Cdk5rap3 knockout (CKO) liver sections. C: Expression of CDK5RAP3 in WT and CKO livers assessed by using Western blot analysis. D: Liver-to-body weight ratio (LW/BW) of CKO and WT mice at 0, 24, 36, and 48 hours after PHx. E: Serum alanine aminotransferase (ALT) levels in CKO and WT mice at 24, 36, and 48 hours after PHx. F and G: Expression of $\beta$-catenin, cyclin D1, matrix metallopeptidase 2 (MMP2), and P21 in WT and CK0 livers assessed by using Western blot analysis at 24, 36, and 48 hours after PHx. Data are expressed as means \pm SD (D and E). Representative results from three experiments (A, C, F, and $\mathbf{G}) . n \geq 4$ in each group (D and E). ${ }^{*} P<0.05,{ }^{* *} P<0.0005$. Scale bars $=100 \mu \mathrm{m}$. GAPDH, glyceraldehyde-3phosphate dehydrogenase.

number of pHH3-labeled cells was significantly decreased in CKO mice (Figure 2D). Moreover, at 48 hours after PHx, PCNA- and BrdU-labeled cell numbers in CKO mice reached levels comparable to that found at 24 hours after PHx in WT control mice (Figure 2, B and $\mathrm{C}$ ). Taken together, these results suggest that $\mathrm{PHx}-$ induced liver regeneration is significantly delayed in CKO mice. 
A
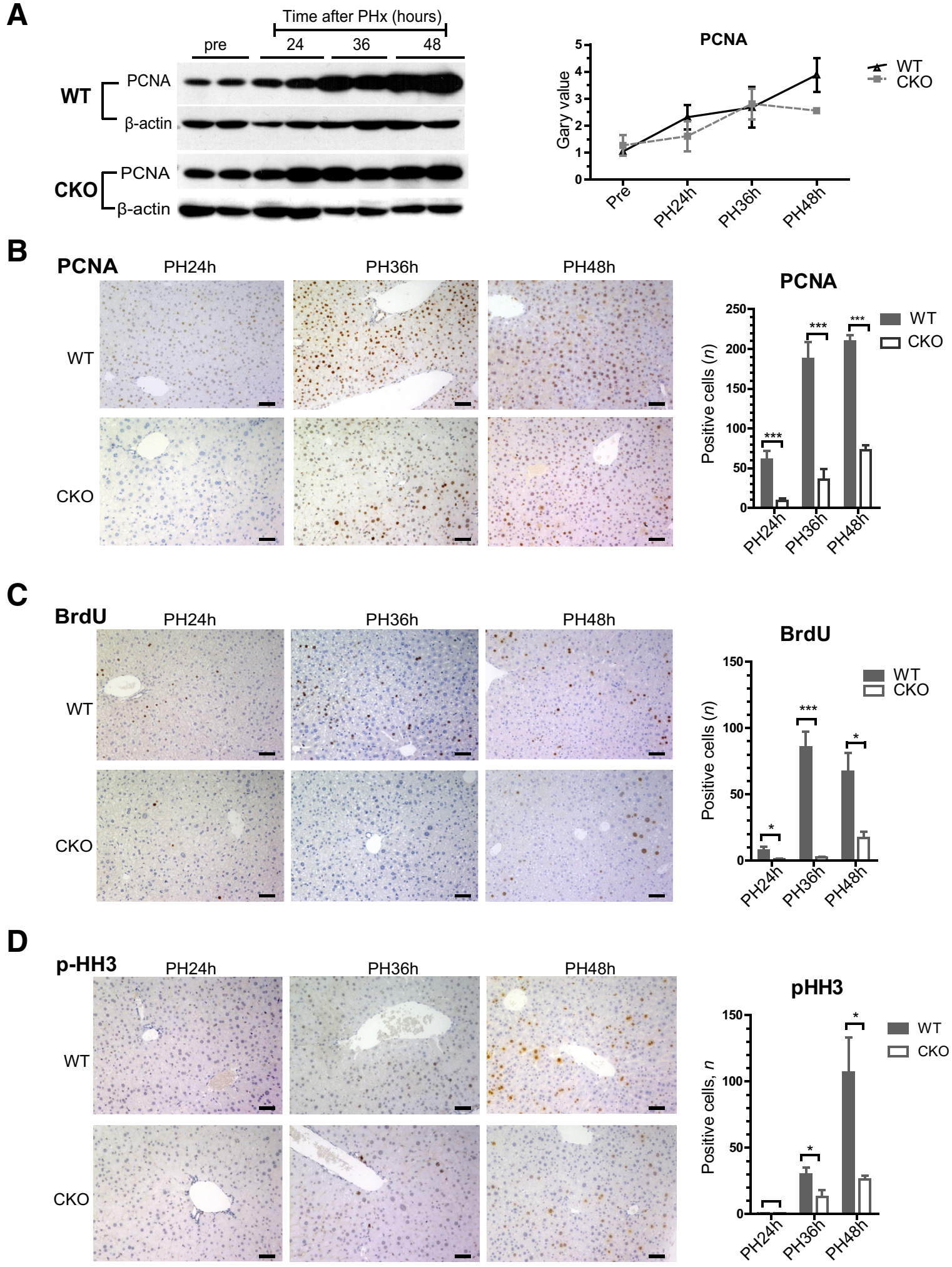

Figure 2 The disruption of CDK5 regulatory subunit-associated protein 3 (CDK5RAP3) in hepatocytes inhibits cell proliferation after partial hepatectomy (PHx). A: Western blot analysis of proliferation cell nuclear antigen (PCNA) protein expression in liver-specific Cdk5rap3 knockout (CK0) and wild-type (WT) livers at $\mathbf{0}, 24,36$, and 48 hours after PHx. Quantification of Western blot results is normalized to $\beta$-actin level. B-D: Immunohistochemistry with PCNA (B) and bromodeoxyuridine (BrdU) incorporation (C), and pHH3 (D) at 24, 36, and 48 hours after PHx in WT and CKO liver sections. The numbers of PCNA-positive (B), BrdU-positive (C), and pHH3-positive (D) hepatocytes are quantified from CKO and WT liver sections at 24, 36, and 48 hours after PHx. Data are expressed as means \pm SD. $n=9$ in each group $(\mathbf{B}-\mathbf{D})$. ${ }^{*} P<0.05,{ }^{* * *} P<0.0005$. Scale bars $=50 \mu \mathrm{m}$ (B-D). 
A

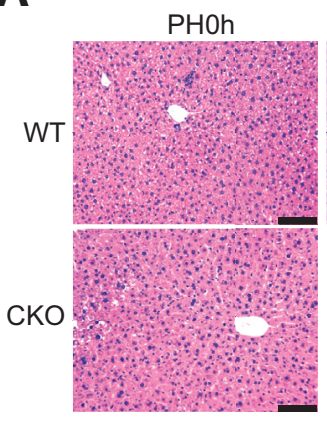

$\mathrm{PH} 24 \mathrm{~h}$
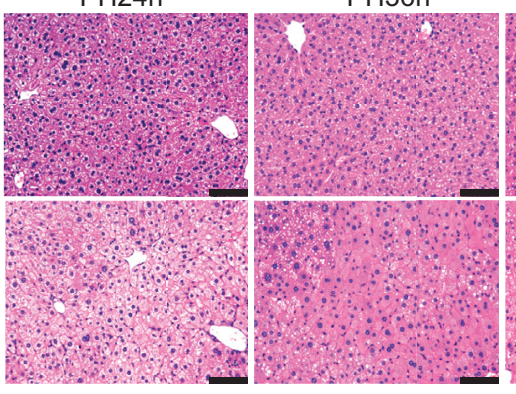

C

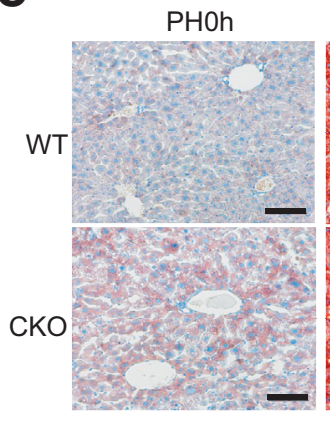

$\mathrm{PH} 24 \mathrm{~h}$

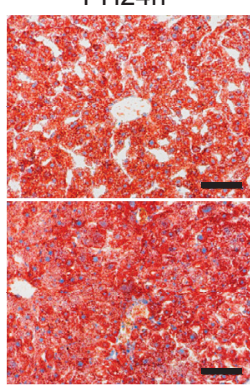

PH36h

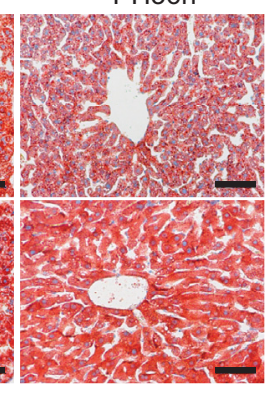

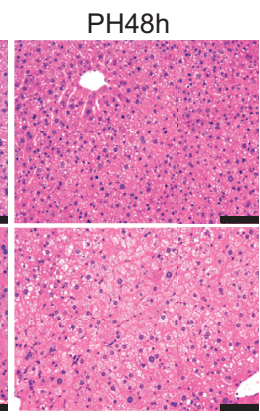

B

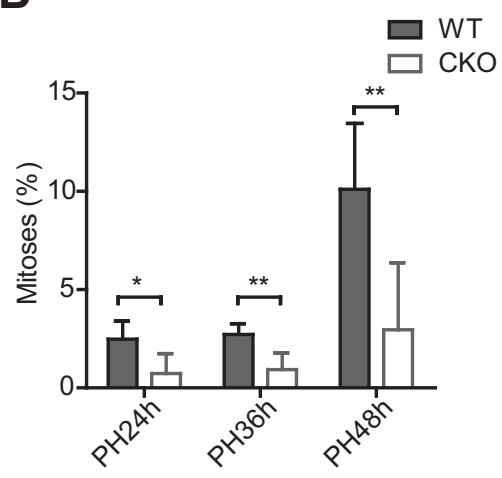

D PH48h
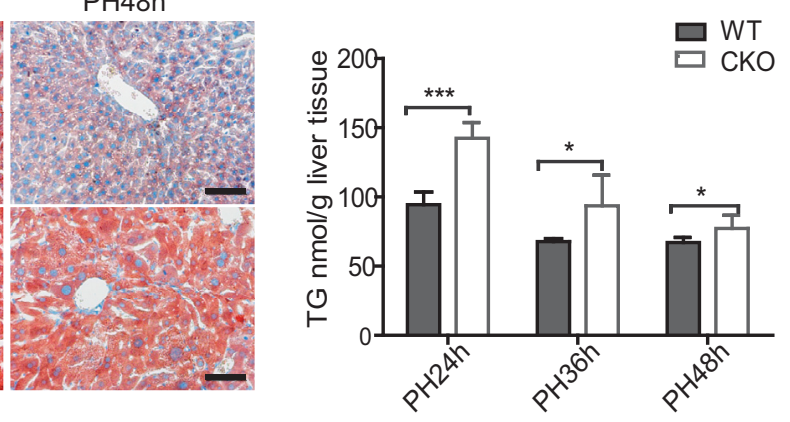

E

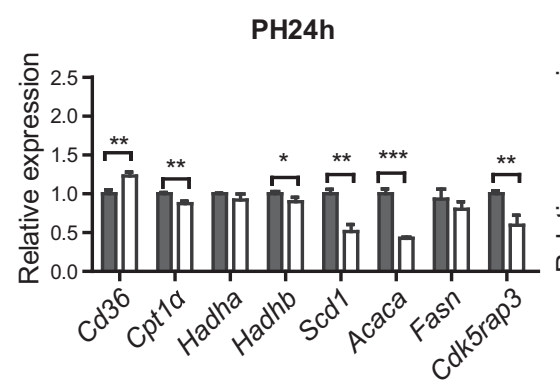

F

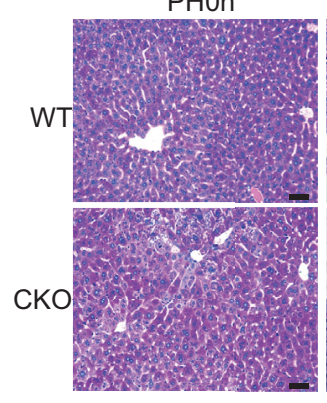

$\mathrm{PH} 24 \mathrm{~h}$

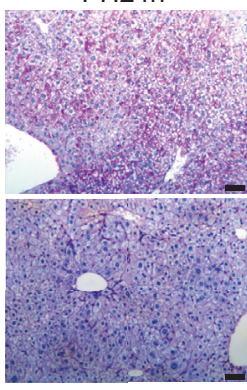

$\mathrm{PH} 36 \mathrm{~h}$

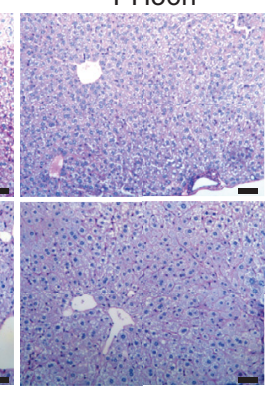

PH36h

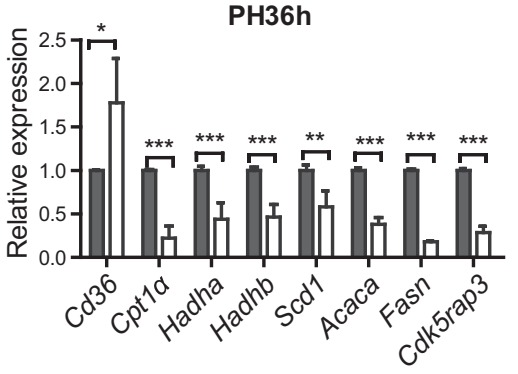

$\mathrm{PH} 48 \mathrm{~h}$

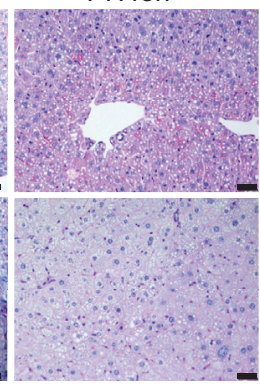

G

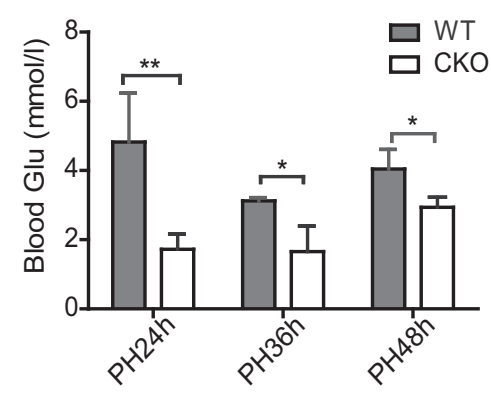

Figure 3 Hepatic CDK5 regulatory subunit-associated protein 3 (CDK5RAP3) deficiency promotes hepatic lipid accumulation and decreases glycogen synthesis during liver regeneration. A: Representative images of hematoxylin and eosin staining from wild-type (WT) and liver-specific Cdk5rap 3 knockout (CK0) liver sections at 0, 24, 36, and 48 hours after partial hepatectomy (PHx). B: The number of mitotic hepatocytes was quantified in CKO and WT mice at 24, 36, and 48 hours after PHx. C: Representative images of Oil Red 0 staining of liver tissues from CKO and WT mice at 0, 24, 36, and 48 hours after PHx. D: Hepatic triglycerides (TG) level in CKO and WT liver tissues at 24 , 36, and 48 hours after PHx. E: Hepatic gene expression for Cd36 (lipid import), Cpt1a, Hadha, Hadhb ( $\beta$-oxidation), and Scd1, Acaca, and Fasn (lipogenesis). $\beta$-actin expression was used to normalize gene expression. F: Representative images of periodic acid-Schiff staining in WT and CKO livers at 0, 24, 36, and 48 hours after PHx. G: Serum glucose (Glu) levels of adult CKO and WT mice. Data are expressed as means \pm SD (B and D-F). $n=5$ in each group (B); $n=3$ to 6 (D); $n=3$ to 4 (G). * $P<0.05$, ${ }^{* *} P<0.005,{ }^{* * *} P<0.0005$. Scale bars: $50 \mu \mathrm{m}$ (A and $\left.\mathbf{F}\right) ; 100 \mu \mathrm{m}(\mathbf{C})$. 


\section{A}

PH7d

HE
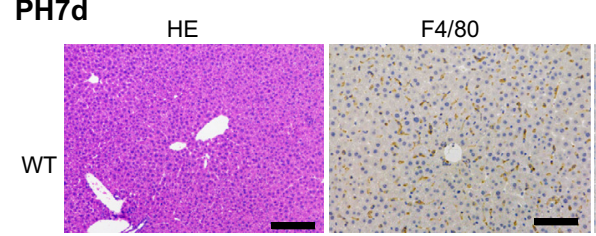

Active

caspase-3

PAS

Oil Red O

CDK5RAP3

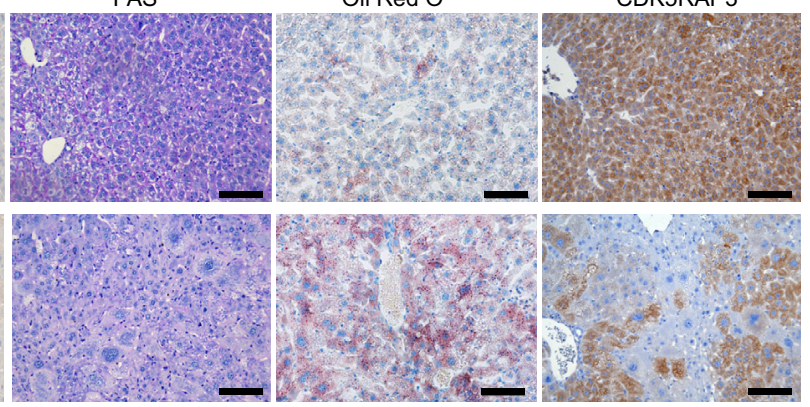

B

PH7d
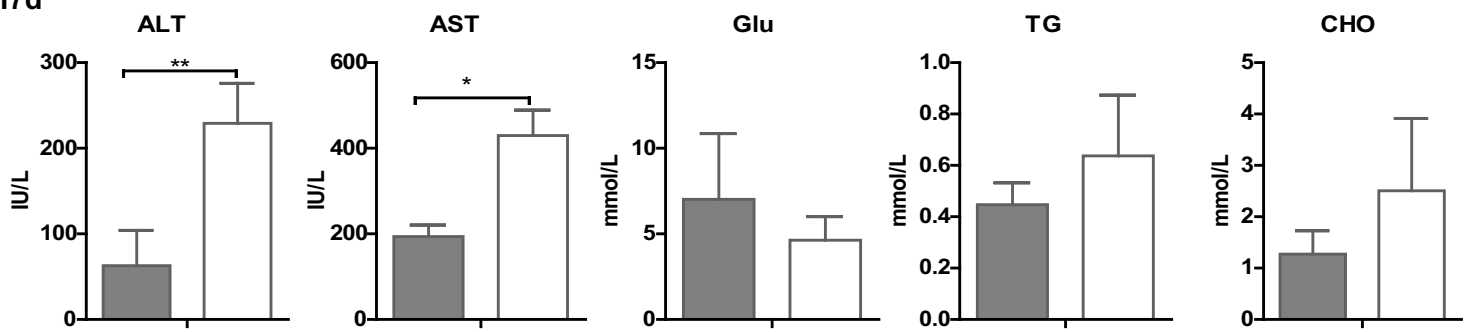

C

PH14d

H\&E

$\mathrm{F} 4 / 80$

Active
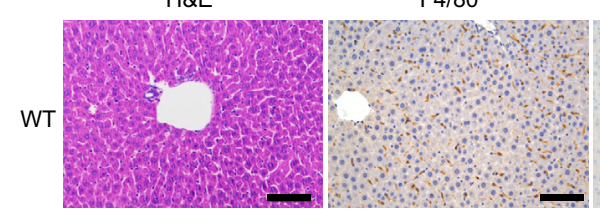

caspase-3

PAS

Oil Red O

CDK5RAP3

$\square$ WT

$\square \mathrm{CKO}$
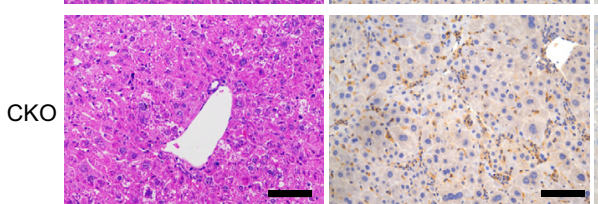

D

\section{PH14d}

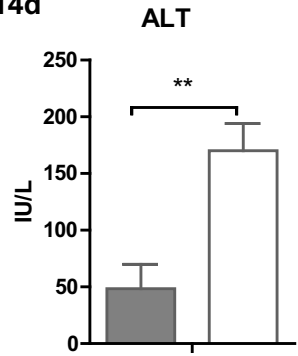

AST

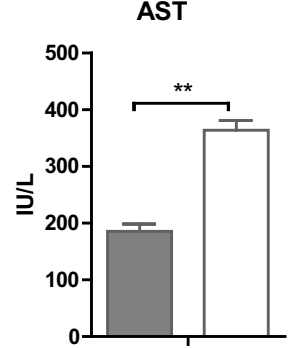

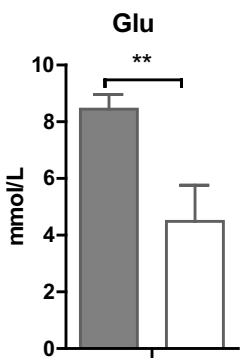
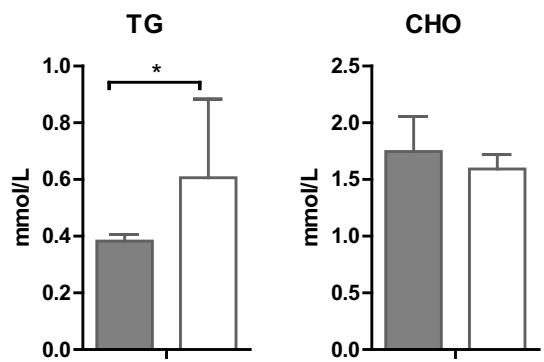

$\square$ WT

Figure 4 Hepatic CDK5 regulatory subunit-associated protein 3 (CDK5RAP3) deficiency leads to exacerbation of inflammatory response and hepatocyte apoptosis during liver regeneration. Representative images of hematoxylin and eosin (H\&E) staining, Oil Red 0 staining, immunohistochemistry for F4/80, periodic acid-Schiff (PAS) staining, immunohistochemistry for active caspase-3, and CDK5RAP3 of wild-type (WT) and liver-specific Cdk5rap3 knockout (CK0) liver sections at $7(\mathbf{A})$ and $14(\mathbf{C})$ days after partial hepatectomy $(\mathrm{PHx})$. Serum alanine aminotransferase (ALT), aspartate aminotransferase (AST), glucose (Glu), triglycerides (TG), and cholesterol (CHO) levels in CKO and control mice at 7 (B) and 14 (D) days after PHx. Data are expressed as means \pm SD. $n=3$ (A-D). ${ }^{*} P<0.05,{ }^{* *} P<0.005$. Scale bars $=50 \mu \mathrm{m}$. 
A
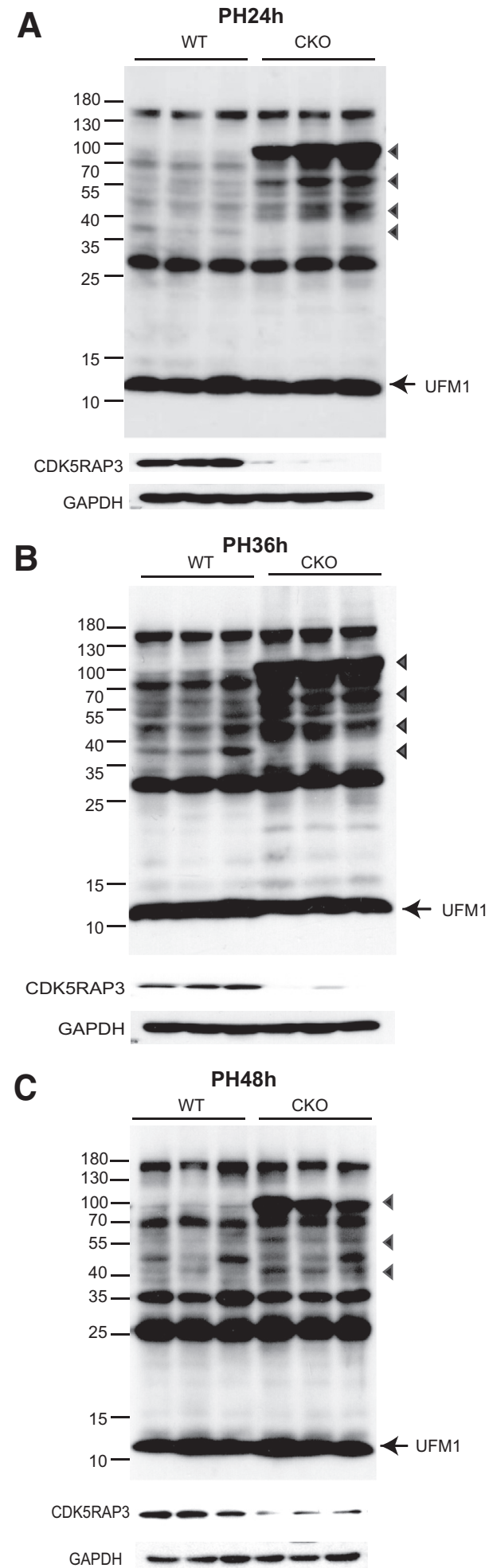

Figure 5 Absence of $C D K 5$ regulatory subunit-associated protein 3 (CDK5RAP3) alters UFMylated substrates in regenerating liver. Ubiquitin-fold modifier 1 (UFM1) conjugates in adult liver-specific Cdk5rap3 knockout (CKO) and control mice were detected by Western blot using anti-UFM1 antibodies at 24 (A), 36 (B), and 48 (C) hours after partial hepatectomy $(\mathrm{PHx})$. Triangles indicate the bands with significant differences. Representative results of three experiments. GAPDH, glyceraldehyde-3-phosphate dehydrogenase.
CDK5RAP3 Deficiency Causes Hepatic Lipid Overload and Impairs Glucose Metabolism during Liver Regeneration

To evaluate the histologic changes occurring during liver regeneration, H\&E staining on liver sections taken from CKO and WT mice was performed at various times after PHx. The organization of the hepatocytes was disrupted in CKO mice compared with WT mice (Figure 3A). Moreover, hepatocyte replication was evaluated by quantifying mitotic figures. After PHx, fewer hepatocytes underwent mitosis in CKO mice than in WT mice (Figure 3B). There were no significant histologic charges in WT and CKO mice before and after the sham operation (Supplemental Figure S1E). The size of the hepatocytes increased after two-thirds PHx, peaked at 24 hours, and then gradually decreased. ${ }^{38}$ Nevertheless, it was noted that at 36 and 48 hours after $\mathrm{PHx}$, the hepatocytes of CKO mice were bigger and with larger cytoplasm than those of WT mice (Figure 3A). Based on these results, an onset of steatosis was suspected in CKO mice after PHx. Oil Red O staining was used to visualize hepatic lipid accumulation. Expectedly, more lipid droplets were found in CKO mice at 24, 36, and 48 hours after PHx (Figure 3C). Consistent with this finding, the amount of liver triglycerides was markedly higher in CKO mice (Figure 3D). Moreover, lipid metabolism was monitored at different time points after PHx. The expression of the lipid import gene $C d 36$ was markedly increased in CKO mice at 24, 36, and 48 hours after PHx. By contrast, Cdk5rap3 deletion caused decreased expression of $\beta$-oxidation genes (Cptl $\alpha$ and Hadha/b) and lipogenic genes (Scadl, Acaca, and Fasn) after PHx (Figure 3E). In addition to lipid oxidation, glucose metabolism provides energy for liver regeneration. ${ }^{39,40}$ Liver glycogen accumulation and blood glucose levels were therefore measured after PHx. Periodic acid-Schiff staining showed that glycogen storage was strongly disrupted in CKO livers compared with WT livers (Figure 3F). Glucose levels in the blood of CKO mice were significantly lower than in the control mice (Figure 3G). These data suggest that CDK5RAP3 is involved in energy supply for liver regeneration by regulating lipid accumulation and glucose metabolism.

\section{CDK5RAP3 Deletion Intensifies Inflammation and Induces Apoptosis at Late-Stage Liver Regeneration}

In the two-thirds PHx rodent model, the remaining liver enlarges until the original liver mass is restored, approximately 1 week after surgery, after which the regenerative process stops. ${ }^{14}$ The effect of CDK5RAP3 deficiency was thus further examined on late-stage liver regeneration. As indicated by their heterogeneous size visualized by $\mathrm{H} \& \mathrm{E}$ staining, the arrangement of hepatocytes was strongly disorganized in CKO mice at 7 and 14 days after PHx, compared with WT mice (Figure 4, A and C). Next, the inflammatory cell infiltrates in WT and CKO livers were 
A

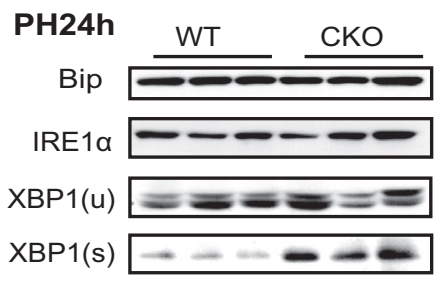

B
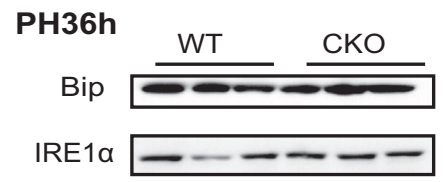

$\mathrm{XBP} 1(\mathrm{u}) \mathrm{Sz}$

$\mathrm{XBP} 1(\mathrm{~s}) \quad \ldots$

C

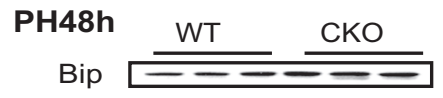

IRE1 ---

XBP1(u) $=$

XBP1(s) $=-\infty$
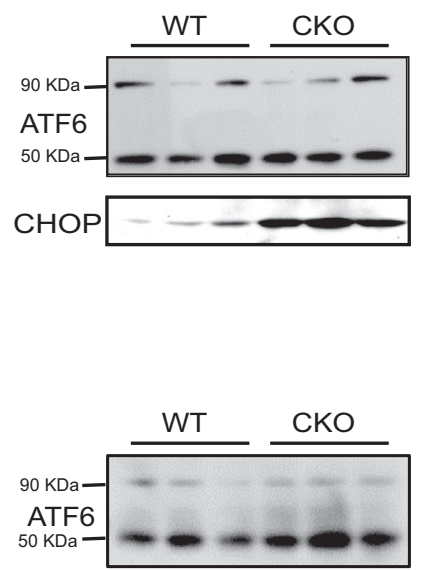

$\mathrm{CHOP}-1-$
p-PERK $\frac{\text { WT }}{2}=$

p-elF2a $=$

ATF4 $\longrightarrow$

CDK5RAP3

GAPDH

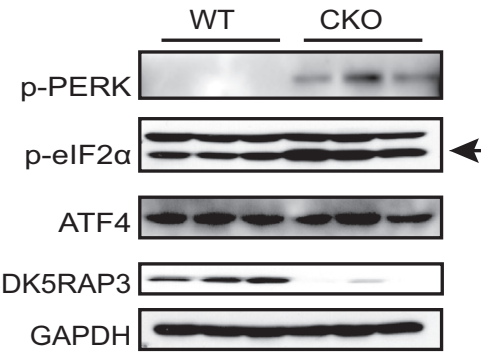

p-PERK $\stackrel{\text { WT }}{-} \stackrel{\text { CKO }}{-\infty-\infty}$

p-elF2 $\alpha=$

ATF4 $---\square$

CDK5RAP3 $\longrightarrow-\ldots$

GAPDH - - -
D

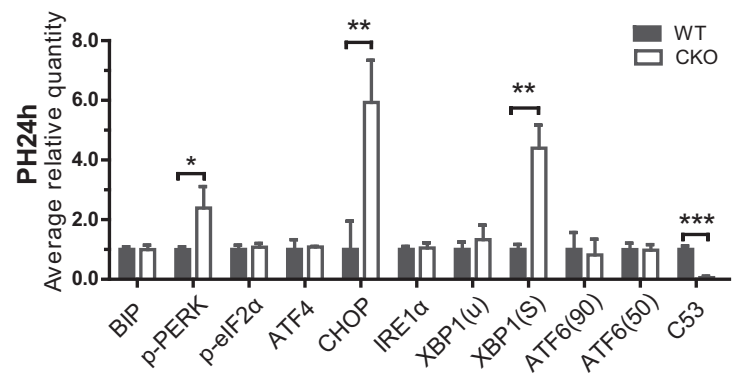

$\mathbf{F}$
E

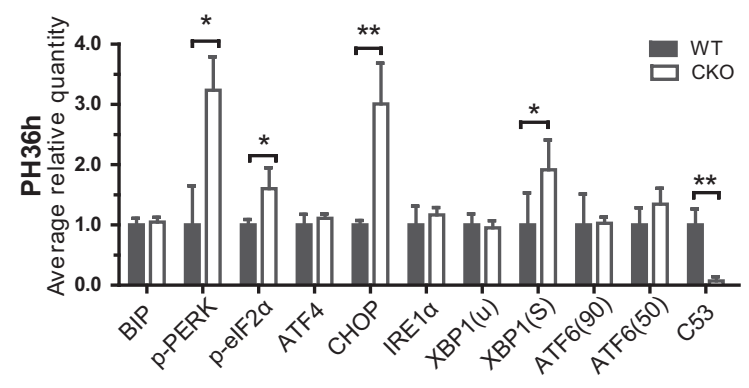

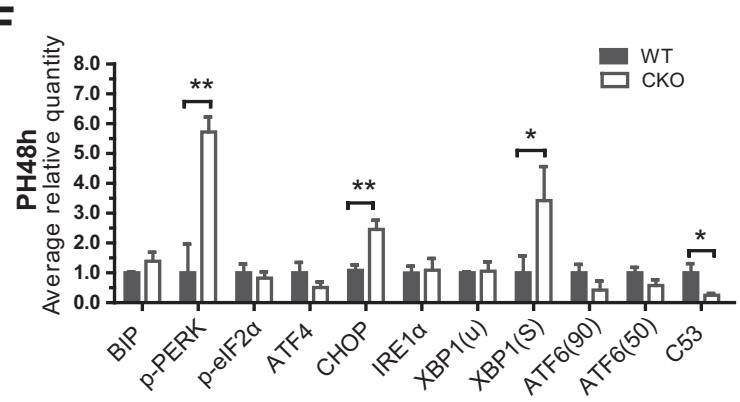

Figure 6 CDK5 regulatory subunit-associated protein 3 (CDK5RAP3) deficiency induces endoplasmic reticulum stress. A-C: Expressions of BiP, p-PERK, p-eIF2 $\alpha$, activating transcription factor (ATF) 4, CCAAT-enhancer-binding protein homologous protein (CHOP), IRE1 $\alpha$, unspliced XBP-1 [XBP-1(u)] and its spliced forms [XBP-1(s)], and ATF6 were analyzed by Western blot in 2-month-old liver-specific Cdk5rap3 knockout (CKO) and control livers at 24 (A), 36 (B), and 48 (C) hours after partial hepatectomy (PHx). Black arrows indicate the P-eIF2 $\alpha$ target proteins. D-F: Expression of endoplasmic reticulum stress-related proteins was measured according to gray scale values from the Western blot bands presented in Figure 6 . Quantification of Western blot results is all normalized to glyceraldehyde-3-phosphate dehydrogenase (GAPDH) levels. Data are expressed as means $\pm \mathrm{SD}$. ${ }^{*} P<0.05,{ }^{* *} P<0.005,{ }^{* * *} P<0.0005$. 

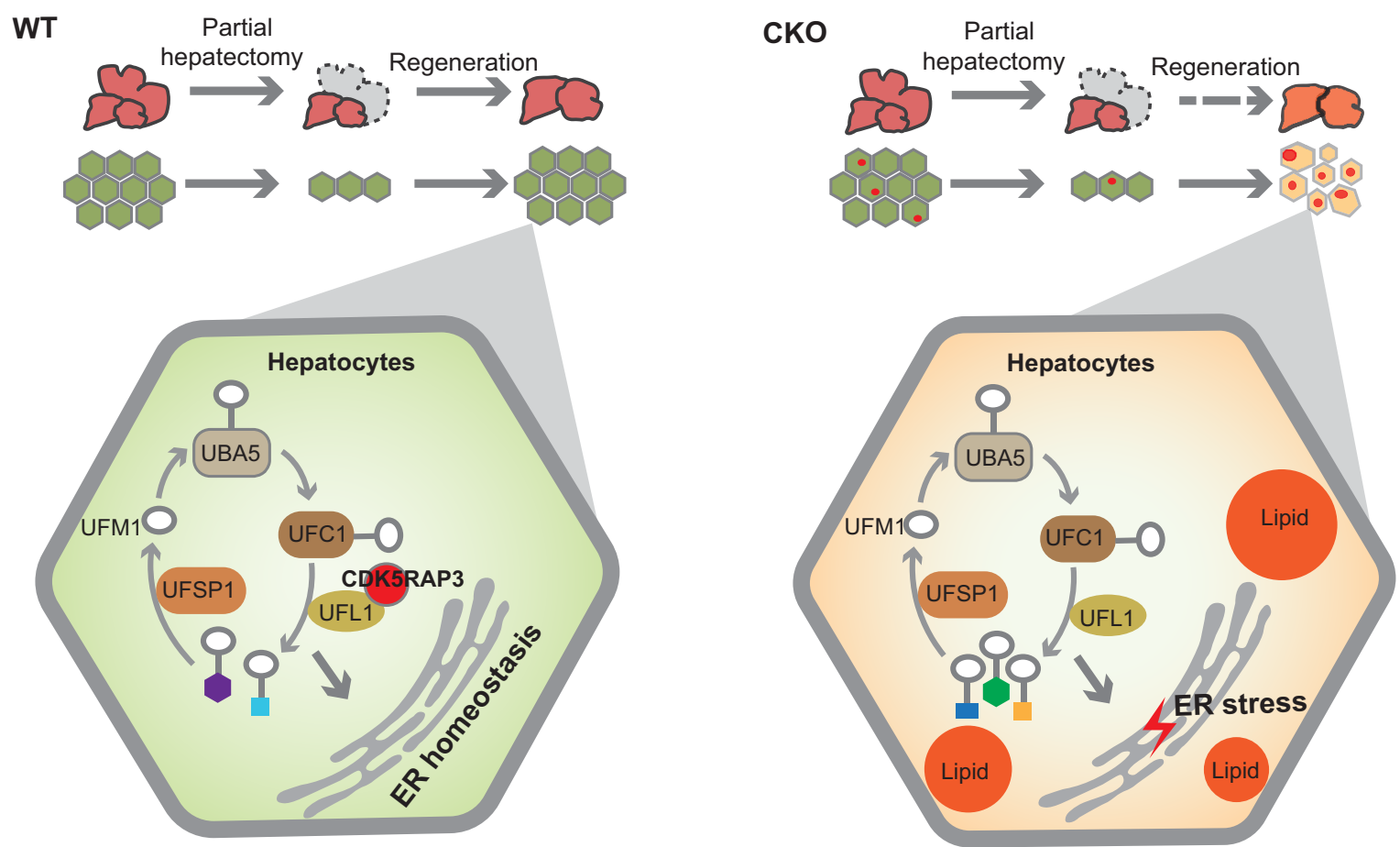

Figure 7 Model for the function of Cdk5rap3 during liver regeneration. In wild-type (WT) mice, CDK5 regulatory subunit-associated protein 3 (CDK5RAP3) controls the substrates specificity of UFMylation to maintain endoplasmic reticulum (ER) homeostasis, sustain normal lipid metabolism, and liver regeneration. In liver-specific Cdk5rap3 knockout (CK0) mice, CDK5RAP3 deficiency leads to altered substrate UFMylation, ER stress induction, impairment of hepatocyte proliferation, aberrant lipid accumulation, and even hepatocyte inflammation and apoptosis. UBA5, E1 activating enzyme; UFC1, E2 conjugating enzyme; UFL1, E3 ligase; UFM1, Ubiquitin-fold modifier 1; UFSP1, and UFSP2, UFM1-specific proteases.

detected (Figure 4, A and C). Consistently, at 7 and 14 days after PHx, there were more F4/80-positive cells in CKO livers than in WT livers (Figure 4, A and C). In addition, evaluation of hepatocytes apoptosis by immunohistochemistry staining for active caspase-3 showed that CDK5RAP3 deficiency induced liver cell apoptosis at 7 and 14 days after PHx (Figure 4, A and C). Day 7 after PHx, lipid accumulation was higher in CKO livers than in WT livers, but no significant difference was visible at day 14 (Figure 4, A and C). Periodic acid-Schiff staining showed that glycogen synthesis was severely disrupted in CKO livers compared with WT livers at 7 and 14 days after PHx.

Serum alanine aminotransferase and aspartate transaminase levels were elevated at 7 and 14 days after PHx; lower serum glucose and higher serum triglycerides were noted in CKO livers compared with WT livers at 14 days after PHx (Figure 4, B and D). The data indicate abnormal CKO liver function at late-stage liver regeneration.

\section{CDK5RAP3 Deficiency Alters UFMylation Profiles in Regenerating Liver}

CDK5RAP3 reportedly interacts with several components of a novel ubiquitin-like modification system, UFMylation. ${ }^{27,32,33,41,42}$ In our previous study, CDK5RAP3 had been recognized as a novel substrate adaptor for
UFMylation. ${ }^{20}$ Whether the aberrant liver regeneration in CKO was accompanied by an alteration of UFMylated substrates has been explored. Intriguingly, the level of UFMylated substrates appeared down-regulated in WT mice after PHx, compared with the homeostatic state (Supplemental Figure S1F). However, CKO mice exhibited the opposite change of UFMylation level after PHx (Supplemental Figure S1F). At the same time, the UFMylation profile in regenerating liver was dramatically altered in CKO mice compared with control mice at 24, 36, and 48 hours after PHx (Figure 5). UFMylated proteins with molecular weights around $100 \mathrm{KDa}$, $55 \mathrm{KDa}$, and $40 \mathrm{KDa}$ were especially up-regulated in CKO mice (Figure 5). In addition, no UFMylated proteins with a molecular weight ranging from $35 \mathrm{KDa}$ to $40 \mathrm{KDa}$ were detected in those CKO mice with higher Cdk5rap3 knockout efficiency (Figure 5). Together, these results indicate that CDK5RAP3-mediated UFMylation may also play important roles in mammalian liver regeneration.

\section{CDK5RAP3 Loss Disturbs ER Homeostasis in Regenerating Liver}

ER stress is associated with liver diseases involving hepatic lipid and glucose metabolism, including deficient regeneration. ${ }^{17,43}$ In recent years, ER homeostasis has been 
associated with steatosis and DNA replication occurring after PHx. ${ }^{17,44}$ Therefore, whether CDK5RAP3 loss disturbed ER homeostasis was immediately investigated in regenerating liver. The expression of proteins involved in three unfolded protein response pathways was analyzed by using Western blot. From the PERK signaling pathway, the phosphorylation level of both PERK and CCAAT-enhancerbinding protein homologous protein was up-regulated at 24 , 36, and 48 hours after PHx (Figure 6), and the phosphorylated form of eIF $2 \alpha$ was only slightly increased at 36 hours after PHx (Figure 6). Activating transcription factor 4 expression showed no change in CKO mice (Figure 6). From the IRE1 $\alpha$ signaling pathway, up-regulation of spliced XBP1 was detected in CKO mice after PHx (Figure 6). Levels of IRE1 $\alpha$ and unspliced XBP1 remained unchanged (Figure 6). In the same way, changes in activating transcription factor 6 expression were not detected in CKO mice after PHx (Figure 6). In summary, the up-regulation of p-PERK, CCAAT-enhancer-binding protein homologous protein, and spliced XBP1 at different time points after PHx shows that CDK5RAP3 loss activated PERK and IRE1 $\alpha$ signaling pathways, thereby increasing ER stress (Figure 6).

Based on our data, a mechanism involving CDK5RAP3 in liver regeneration was proposed (Figure 7). In WT mice, CDK5RAP3 controls the substrate specificity of UFMylation to maintain ER homeostasis, sustain normal lipid metabolism, and promote liver regeneration. Although CDK5RAP3 deficiency does not influence the priming of liver regeneration, it leads to altered substrate UFMylation, ER stress induction, impairment of hepatocyte proliferation, aberrant lipid accumulation, and even hepatocyte inflammation and apoptosis.

\section{Discussion}

PHx is increasingly used to manage diverse malignant and nonmalignant lesions. ${ }^{45}$ The regenerative capacity of the liver is crucial for survival of patients undergoing partial resection and after acute and chronic liver injuries secondary to toxins, infections, immune dysfunction, or metabolic diseases. ${ }^{5,12}$ Two-thirds PHx is widely used to assess liver regeneration capacity in rodent models. ${ }^{46}$ This study showed that CDK5RAP3 deficiency impairs liver regeneration through inhibiting hepatocyte proliferation and affecting lipid and glucose metabolism. CDK5RAP3 deletion induced inflammatory response and even liver apoptosis at a late stage of liver regeneration. Alteration of UFMylation profile and induction of ER stress response occurring upon CDK5RAP3 deficiency likely explain the impaired liver regeneration. Collectively, these results indicate that CDK5RAP3 plays a significant role in liver regeneration.

Lipid oxidation and glucose metabolism are reported as the primary energy source early after hepatectomy. ${ }^{15,47}$ Lipids are expended as energy for hepatocyte DNA replication and as materials for phospholipid synthesis. ${ }^{48,49}$ The liver has a robust and unique regenerative capacity in response to damage, ${ }^{14}$ but this capacity is suppressed in hepatic steatosis. ${ }^{46}$ Impaired fatty liver regeneration is a leading cause of postoperative complications after hepatectomy. ${ }^{50-52}$ Fatty liver has been recognized as a significant risk factor for defective liver regeneration in humans and animal models. ${ }^{40}$ However, a transient hepatocellular lipid accumulation characteristic of early regeneration after $\mathrm{PHx}$ in rodents is virtually required for physiological liver regeneration. ${ }^{40}$ Transient liver steatosis at 12 to 24 hours after PHx is crucial for normal liver regeneration, but excess hepatic steatosis and disturbance of hepatic lipid accumulation before PHx suppress liver regeneration in mice. ${ }^{53}$ Posthepatectomy recovery is impaired in fatty liver, characterized by both a decrease in hepatocyte proliferation and an increase in cell death. ${ }^{54}$ In this study, CKO mice displayed no obvious defects and survived well into adulthood. However, homeostatic lipid storage in adult CKO mice is up-regulated, indicating that $\mathrm{CKO}$ mice may exhibit abnormal lipid metabolism. In addition, CDK5RAP3 deficiency in liver resulted in excessive hepatic lipid accumulation and reduced hepatocyte proliferation after $\mathrm{PHx}$, which may contribute to impaired liver regeneration. Through expression analysis of genes involved in lipid metabolism, lipid import was significantly up-regulated in CKO mice. However, the $\beta$-oxidation and lipogenesis were impaired in the absence of CDK5RAP3. Hence, we speculated that CDK5RAP3 deletion did not affect initiation of transient liver steatosis but impaired the lipid consumption. Apart from lipids, a large amount of stored glycogen provides an energy reserve useable for hepatocytes' DNA replication in case of PHx. ${ }^{8}$ In this study, the lower glycogen synthesis observed in Cdk5rap3 mutants might be the cause of proliferation delay. Blood glucose level reflects the liver mass, which may act as a sensor after PHx. ${ }^{8}$ Hypoglycemia and increased circulating insulin immediately follow PHx. ${ }^{15,55}$ The shift from glucose to lipid consumption is significant for the regeneration of phosphatase and tensin homolog-deficient livers after PHx. ${ }^{47}$ In this study, blood glucose levels were decreased in CKO mice. Whether this difference affects steatosis and hepatocyte proliferation is worthy of concern. From our study, we propose that some liver functions, such as regulation of glucolipid metabolism and damage response, may be defective in CKO mice. Part of this hypothesis can be addressed by feeding CKO mice a high-fat diet after liver regeneration to verify the function of the regenerative liver.

Proteins involved in ubiquitination and proteasomal degradation were highly up-regulated after PHx versus sham surgery. ${ }^{56}$ The E3 ubiquitin-protein ligase UHRF1 increases during liver regeneration independently of BIRC5. ${ }^{57}$ Another ubiquitin ligase, Nedd4-1, was reported as a crucial regulator of liver regeneration. ${ }^{56}$ Deletion of HOIP, which is the catalytic subunit of the linear ubiquitin chain assembly complex, promotes liver regeneration and DNA 
damage. ${ }^{58}$ However, thus far, the role of UFMylation, a novel ubiquitin-like modification system, in mammalian liver regeneration was unknown. Previous studies showed the interactions between CDK5RAP3 and multiple UFMylation-related factors. ${ }^{32,41,59}$ Moreover, we have identified CDK5RAP3 as a novel substrate adaptor for this ubiquitin-like modification. ${ }^{20}$ This study showed that CDK5RAP3 deficiency also influences the UFMylation profile in liver after PHx. Meanwhile, fluctuation of UFMylation level was detected in some proteins at different time points after PHx. To our knowledge, this report is the first regarding the function of UFMylation in liver regeneration.

Liver regeneration after PHx enhances the unfolded protein burden at the ER of remnant hepatocytes, which in turn triggers the unfolded protein response. ${ }^{45}$ ER stress plays an important role in cell fate control, including cell cycle, metabolism, and lipid synthesis. ${ }^{60}$ In this study, depletion of CDK5RAP3 triggered ER stress and activated unfolded protein response after $\mathrm{PHx}$, through the upregulation of phosphorylation of PERK, spliced XBP-1, and CCAAT-enhancer-binding protein homologous protein. XBP-1 is induced by $\mathrm{PHx}$ and modulates unfolded protein, acute-phase, and DNA damage responses during mouse liver regeneration. ${ }^{45}$ Thus, XBP-1 may mediate the effect of CDK5RAP3 defect on impaired liver regeneration. However, many components of the UFM1 system regulate ER homeostasis. ${ }^{31,61}$ The disturbance of UFMylation mediated by CDK5RAP3 may result in exacerbated ER stress after PHx.

Nonalcoholic fatty liver disease is a liver metabolic syndrome associated with insulin resistance and dyslipidemia. ${ }^{62}$ Fatty liver has been identified as a major risk factor for defective liver regeneration. ${ }^{17}$ ER stress is involved in hepatic lipid and glucose metabolism in liver diseases, including deficient regeneration. ${ }^{43}$ In the current study, we found that Cdk5rap3 deficiency not only increases homeostatic lipid accumulation but also prominently intensifies steatosis after PHx, at least partially through exacerbation of ER stress. In conclusion, CDK5RAP3 constitutes a potential therapeutic target in the treatment of patients with nonalcoholic fatty liver disease and aberrant regeneration.

\section{Acknowledgment}

We thank Dr. Houzao Chen for providing the PHx experiment design and operation protocol.

\section{Author Contributions}

Y.H., Y.J., and S.Y. wrote the original draft; Y.H., Y.J., S.Y., and R.Y. reviewed and edited the manuscript; Y.H. and Y.J. supervised the work; Y.H. and Y.J. provided administration for the project; Y.H. and Y.J. acquired funding; all authors contributed to investigation and data curation.

\section{Supplemental Data}

Supplemental material for this article can be found at http://doi.org/10.1016/j.ajpath.2020.08.011.

\section{References}

1. Palmes D, Spiegel HU: Animal models of liver regeneration. Biomaterials 2004, 25:1601-1611

2. Alison MR, Islam S, Lim S: Stem cells in liver regeneration, fibrosis and cancer: the good, the bad and the ugly. J Pathol 2009, 217: 282-298

3. Lisman T, Porte RJ: Mechanisms of platelet-mediated liver regeneration. Blood 2016, 128:625-629

4. Rahnemai-Azar AA, Cloyd JM, Weber SM, Dillhoff M, Schmidt C, Winslow ER, Pawlik TM: Update on liver failure following hepatic resection: strategies for prediction and avoidance of post-operative liver insufficiency. J Clin Transl Hepatol 2018, 6:97-104

5. Michalopoulos GK: Liver regeneration. J Cell Physiol 2007, 213: 286-300

6. Su AI, Guidotti LG, Pezacki JP, Chisari FV, Schultz PG: Gene expression during the priming phase of liver regeneration after partial hepatectomy in mice. Proc Natl Acad Sci U S A 2002, 99: $11181-11186$

7. Xie G, Yin S, Zhang Z, Qi D, Wang X, Kim D, Yagai T, Brocker CN, Wang Y, Gonzalez FJ, Wang H, Qu A: Hepatocyte peroxisome proliferator-activated receptor alpha enhances liver regeneration after partial hepatectomy in mice. Am J Pathol 2019, 189:272-282

8. Miyaoka Y, Miyajima A: To divide or not to divide: revisiting liver regeneration. Cell Division 2013, 8:8

9. Michalopoulos GK: Liver regeneration after partial hepatectomy: critical analysis of mechanistic dilemmas. Am J Pathol 2010, 176:2-13

10. Ezquer F, Bahamonde J, Huang YL, Ezquer M: Administration of multipotent mesenchymal stromal cells restores liver regeneration and improves liver function in obese mice with hepatic steatosis after partial hepatectomy. Stem Cell Res Ther 2017, 8:20

11. Kwon YJ, Lee KG, Choi D: Clinical implications of advances in liver regeneration. Clin Mol Hepatol 2015, 21:7-13

12. Fausto N, Campbell JS, Riehle KJ: Liver regeneration. Hepatology 2006, 43 Suppl 1:S45-S53

13. Michalopoulos GK, DeFrances MC: Liver regeneration. Science 1997, 276:60-66

14. Taub R: Liver regeneration: from myth to mechanism. Nat Rev Mol Cell Biol 2004, 5:836-847

15. Huang J, Rudnick DA: Elucidating the metabolic regulation of liver regeneration. Am J Pathol 2014, 184:309-321

16. Siu J, McCall J, Connor S: Systematic review of pathophysiological changes following hepatic resection. HPB (Oxford) 2014, 16:407-421

17. Hamano M, Ezaki H, Kiso S, Furuta K, Egawa M, Kizu T, Chatani N, Kamada Y, Yoshida Y, Takehara T: Lipid overloading during liver regeneration causes delayed hepatocyte DNA replication by increasing ER stress in mice with simple hepatic steatosis. J Gastroenterol 2014, 49:305-316

18. Wang X, Ching YP, Lam WH, Qi Z, Zhang M, Wang JH: Identification of a common protein association region in the neuronal Cdk5 activator. J Biol Chem 2000, 275:31763-31769

19. Ching YP, Qi Z, Wang JH: Cloning of three novel neuronal Cdk5 activator binding proteins. Gene 2000, 242:285-294

20. Yang R, Wang H, Kang B, Chen B, Shi Y, Yang S, Sun L, Liu Y, Xiao W, Zhang T, Yang J, Zhang Y, Zhu M, Xu P, Chang Y, Jia Y, 
Huang Y: CDK5RAP3, a UFL1 substrate adaptor, is crucial for liver development. Development 2019, 146:dev169235

21. Wang J, An H, Mayo MW, Baldwin AS, Yarbrough WG: LZAP, a putative tumor suppressor, selectively inhibits NF-kappaB. Cancer Cell 2007, 12:239-251

22. Wang J, He X, Luo Y, Yarbrough WG: A novel ARF-binding protein (LZAP) alters ARF regulation of HDM2. Biochem J 2006, 393(Pt 3): 489-501

23. Jiang $\mathrm{H}$, Luo $\mathrm{S}$, Li H: Cdk5 activator-binding protein $\mathrm{C} 53$ regulates apoptosis induced by genotoxic stress via modulating the G2/M DNA damage checkpoint. J Biol Chem 2005, 280:20651-20659

24. Liu D, Wang WD, Melville DB, Cha YI, Yin Z, Issaeva N, Knapik EW, Yarbrough WG: Tumor suppressor Lzap regulates cell cycle progression, doming, and zebrafish epiboly. Dev Dyn 2011, 240:1613-1625

25. Jiang $\mathrm{H}$, Wu J, He C, Yang W, Li H: Tumor suppressor protein C53 antagonizes checkpoint kinases to promote cyclin-dependent kinase 1 activation. Cell Res 2009, 19:458-468

26. Shiwaku H, Yoshimura N, Tamura T, Sone M, Ogishima S, Watase K, Tagawa K, Okazawa H: Suppression of the novel ER protein Maxer by mutant ataxin-1 in Bergman glia contributes to noncell-autonomous toxicity. EMBO J 2010, 29:2446-2460

27. Kwon J, Cho HJ, Han SH, No JG, Kwon JY, Kim H: A novel LZAPbinding protein, NLBP, inhibits cell invasion. J Biol Chem 2010, 285: $12232-12240$

28. Komatsu M, Chiba T, Tatsumi K, Iemura S, Tanida I, Okazaki N, Ueno T, Kominami E, Natsume T, Tanaka K: A novel proteinconjugating system for Ufm1, a ubiquitin-fold modifier. EMBO J 2004, 23:1977-1986

29. Daniel J, Liebau E: The ufm 1 cascade. Cells 2014, 3:627-638

30. Wei Y, Xu X: UFMylation: a Unique \& Fashionable Modification for Life. Genomics Proteomics Bioinformatics 2016, 14:140-146

31. Gerakis Y, Quintero M, Li H, Hetz C: The UFMylation system in proteostasis and beyond. Trends Cell Biol 2019, 29:974-986

32. Yoo HM, Kang SH, Kim JY, Lee JE, Seong MW, Lee SW, Ka SH, Sou YS, Komatsu M, Tanaka K, Lee ST, Noh DY, Baek SH, Jeon YJ, Chung CH: Modification of ASC1 by UFM1 is crucial for ERalpha transactivation and breast cancer development. Mol Cell 2014, 56:261-274

33. Zhang Y, Zhang M, Wu J, Lei G, Li H: Transcriptional regulation of the Ufm1 conjugation system in response to disturbance of the endoplasmic reticulum homeostasis and inhibition of vesicle trafficking. PLoS One 2012, 7:e48587

34. Cai Y, Pi W, Sivaprakasam S, Zhu X, Zhang M, Chen J, Makala L, Lu C, Wu J, Teng Y, Pace B, Tuan D, Singh N, Li H: UFBP1, a key component of the Ufm1 conjugation system, is essential for Ufmylation-mediated regulation of erythroid development. PLoS Genet 2015, 11:e1005643

35. Lehmann K, Tschuor C, Rickenbacher A, Jang JH, Oberkofler CE, Tschopp O, Schultze SM, Raptis DA, Weber A, Graf R, Humar B, Clavien PA: Liver failure after extended hepatectomy in mice is mediated by a p21-dependent barrier to liver regeneration. Gastroenterology 2012, 143:1609-16019 e4

36. Mitchell C, Willenbring $\mathrm{H}$ : A reproducible and well-tolerated method for $2 / 3$ partial hepatectomy in mice. Nat Protoc 2008, 3:1167-1170

37. Mitchell C, Willenbring H: Addendum: a reproducible and well-tolerated method for 2/3 partial hepatectomy in mice. Nat Protoc 2014, 9:10

38. Miyaoka Y, Ebato K, Kato H, Arakawa S, Shimizu S, Miyajima A: Hypertrophy and unconventional cell division of hepatocytes underlie liver regeneration. Curr Biol 2012, 22:1166-1175

39. Fernández-Rojo MA, Restall C, Ferguson C, Martel N, Martin S, Bosch M, Kassan A, Leong GM, Martin SD, McGee SL, Muscat GE, Anderson RL, Enrich C, Pol A, Parton RG: Caveolin-1 orchestrates the balance between glucose and lipid-dependent energy metabolism: implications for liver regeneration. Hepatology 2012, 55:1574-1584

40. Rudnick DA, Davidson NO: Functional relationships between lipid metabolism and liver regeneration. Int J Hepatol 2012, 2012:549241
41. Lemaire K, Moura RF, Granvik M, Igoillo-Esteve M, Hohmeier HE, Hendrickx N, Newgard CB, Waelkens E, Cnop M, Schuit F: Ubiquitin fold modifier 1 (UFM1) and its target UFBP1 protect pancreatic beta cells from ER stress-induced apoptosis. PLoS One 2011, 6: e18517

42. Wu J, Lei G, Mei M, Tang Y, Li H: A novel C53/LZAP-interacting protein regulates stability of C53/LZAP and DDRGK domaincontaining Protein 1 (DDRGK1) and modulates NF-kappaB signaling. J Biol Chem 2010, 285:15126-15136

43. Bechmann LP, Hannivoort RA, Gerken G, Hotamisligil GS, Trauner M, Canbay A: The interaction of hepatic lipid and glucose metabolism in liver diseases. J Hepatol 2012, 56: 952-964

44. Ben Mosbah I, Alfany-Fernández I, Martel C, Zaouali MA, BintanelMorcillo M, Rimola A, Rodés J, Brenner C, Roselló-Catafau J, Peralta C: Endoplasmic reticulum stress inhibition protects steatotic and non-steatotic livers in partial hepatectomy under ischemiareperfusion. Cell Death Dis 2010, 1:e52

45. Argemí J, Kress TR, Chang HCY, Ferrero R, Bertolo C, Moreno H, González-Aparicio M, Uriarte I, Guembe L, Segura V, HernandezAlcoceba R, Ávila MA, Amati B, Prieto J, Aragón T: X-box binding protein 1 regulates unfolded protein, acute-phase, and DNA damage responses during regeneration of mouse liver. Gastroenterology 2017, 152:1203-1216.e15

46. Inaba $\mathrm{Y}$, Furutani $\mathrm{T}$, Kimura $\mathrm{K}$, Watanabe $\mathrm{H}$, Haga $\mathrm{S}$, Kido $\mathrm{Y}$, Matsumoto M, Yamamoto Y, Harada K, Kaneko S, Oyadomari S, Ozaki M, Kasuga M, Inoue $\mathrm{H}$ : Growth arrest and DNA damageinducible 34 regulates liver regeneration in hepatic steatosis in mice. Hepatology 2015, 61:1343-1356

47. Kachaylo E, Tschuor C, Calo N, Borgeaud N, Ungethum U, Limani P, Piguet AC, Dufour JF, Foti M, Graf R, Clavien PA, Humar B: PTEN down-regulation promotes beta-oxidation to fuel hypertrophic liver growth after hepatectomy in mice. Hepatology 2017, 66:908-921

48. Farrell GC: Probing Prometheus: fat fueling the fire? Hepatology 2004, 40:1252-1255

49. Farley FW, Soriano P, Steffen LS, Dymecki SM: Widespread recombinase expression using FLPeR (flipper) mice. Genesis 2000, $28: 106-110$

50. Veteläinen R, van Vliet A, Gouma DJ, van Gulik TM: Steatosis as a risk factor in liver surgery. Ann Surg 2007, 245:20-30

51. de Meijer VE, Kalish BT, Puder M, Ijzermans JN: Systematic review and meta-analysis of steatosis as a risk factor in major hepatic resection. Br J Surg 2010, 97:1331-1339

52. Truant S, Bouras AF, Petrovai G, Buob D, Ernst O, Boleslawski E, Hebbar M, Pruvot FR: Volumetric gain of the liver after major hepatectomy in obese patients: a case-matched study in 84 patients. Ann Surg 2013, 258:696-704

53. Shteyer E, Liao Y, Muglia LJ, Hruz PW, Rudnick DA: Disruption of hepatic adipogenesis is associated with impaired liver regeneration in mice. Hepatology 2004, 40:1322-1332

54. Veteläinen R, van Vliet AK, van Gulik TM: Severe steatosis increases hepatocellular injury and impairs liver regeneration in a rat model of partial hepatectomy. Ann Surg 2007, 245:44-50

55. Huang J, Schriefer AE, Cliften PF, Dietzen D, Kulkarni S, Sing S, Monga SP, Rudnick DA: Postponing the Hypoglycemic Response to Partial Hepatectomy Delays Mouse Liver Regeneration. Am J Pathol 2016, 186:587-599

56. Bachofner M, Speicher T, Bogorad RL, Muzumdar S, Derrer CP, Hurlimann F, Bohm F, Nanni P, Kockmann T, Kachaylo E, Meyer M, Padrissa-Altes S, Graf R, Anderson DG, Koteliansky V, Auf dem Keller U, Werner S: Large-scale quantitative proteomics identifies the ubiquitin ligase Nedd4-1 as an essential regulator of liver regeneration. Dev Cell 2017, 42:616-625.e8

57. Bracht $T$, Hagemann S, Loscha M, Megger DA, Padden J, Eisenacher M, Kuhlmann K, Meyer HE, Baba HA, Sitek B: Proteome analysis of a hepatocyte-specific BIRC5 (survivin)-knockout mouse 
model during liver regeneration. J Proteome Res 2014, 13: $2771-2782$

58. Shimizu Y, Peltzer N, Sevko A, Lafont E, Sarr A, Draberova H, Walczak H: The linear ubiquitin chain assembly complex acts as a liver tumor suppressor and inhibits hepatocyte apoptosis and hepatitis. Hepatology 2017, 65:1963-1978

59. Walczak CP, Leto DE, Zhang L, Riepe C, Muller RY, DaRosa PA, Ingolia NT, Elias JE, Kopito RR: Ribosomal protein RPL26 is the principal target of UFMylation. Proc Natl Acad Sci U S A 2019, 116: 1299-1308
60. Hetz C, Papa FR: The unfolded protein response and cell fate control. Mol Cell 2018, 69:169-181

61. Xie Z, Fang Z, Pan Z: Ufl1/RCAD, a Ufm1 E3 ligase, has an intricate connection with ER stress. Int J Biol Macromol 2019, 135:760-767

62. Speliotes EK, Massaro JM, Hoffmann U, Vasan RS, Meigs JB, Sahani DV, Hirschhorn JN, O'Donnell CJ, Fox CS: Fatty liver is associated with dyslipidemia and dysglycemia independent of visceral fat: the Framingham Heart Study. Hepatology 2010, 51: 1979-1987 